\title{
A comparison of two discrimination-reversal indices intended for use with diverse groups of organisms
}

\author{
DUANE M. RUMBAUGH ${ }^{1}$, SAN DIEGO STATE COLLEGE AND INSTITUTE FOR COMPARATIVE BIOLOGY
} MALCOLM A. JEEVES, UNIVERSITY OF ADELAIDE, SOUTH AUSTRALIA

Two discrimination-reversal indices, independently conceived by the authors, are compared and contrasted. The indices avoid many of the complications involved in the method of directly comparing absolute performance levels of diverse species. Each index provides a ratio score which relates reversal performance to acquisition performance on visual discrimination tasks.

The purpose of this paper is to compare two indices of discrimination-reversal ability intended for use in studies where a sensitive measure of capacity for relatively complex tasks is desired for comparing disparate groups of organisms. In studies of phylogenetic comparison, of ontogenetic development within and between species, and of a wide variety of treatment variables (e.g., early environment, pharmacologic, and brain function) where conventional methods of directly comparing absolute performances are of questionable value (as there are usually reasons to doubt the equivalence of Ss' motivational states or perceptual skills and/or the general equity of the test situation for Ss of diverse species) there is clearly a need for a behavioral index which can circumvent in some manner the formidable problems encountered.

The two indices compared are the reversal index, RI (Rajalakshmi \& Jeeves, 1965) and the reversal/ acquisition ratio, R/A (Rumbaugh \& Pournelle, 1966). These closely-related indices of discrimination-reversal learning were independently conceived, the authors of the indices being unaware not only of their common interests, but of the other's existence. By clarifying points of similarity and difference for these indices, the authors intend to advance a viewpoint and an approach which they believe resolves some of the above mentioned problems.

\section{Points in Common}

1. Both indices (Is) are predicated on the assumption that to the degree Ss are able to exhibit efficient discrimination-reversal skills, they possess increased general problem-solving capacity. In the case of primates and those near them in the evolutionary scale, this means increased capacity for handling complex tasks of the kind where ultimately one-trial learning is possible, as is true with the broad class of learningset training situations (Harlow, 1959).

2. Both Is entail an assessment of reversal performance relative to pre-reversal performance, i.e., acquisition performance. Both Is entail calculation of ratios for purposes of behavioral comparisons. Neither I entails comparison of single, absolute performances, such as number or percentage of responses correct.

3 . Basically, both Is reflect the nature of transfer, positive or negative, between acquisition and reversal trials in a visual discrimination learning situation. Both Is are interpreted in ways which make the resultant ratio increasingly superior as the amount of positive transfer increases from the acquisition to the reversal trials, that transfer providing for the reversal performances being relatively better than the acquisition performance. The reverse of this is also true as negative, rather than positive, transfer occurs.

4. Both Is are advanced by their authors as applicable to performances of diverse animals in disparate training situations. That both Is are relatively economical to obtain as well as sensitive to differences which appear after relatively small amounts of training (compared to the amount of training typically involved in other kinds of tasks, e.g., learning-set, delayed response) are additional points, either stated or implied, which the indices hold in common. Both Is provide for optimal differentiation of performance at an early training state $-R I$ being based on problem 1 and $R / A$ ratios differing maximally where $A \%$ approximates $65-70 \%$. Points of Difference

1. RI is based on criterional acquisition and criterional reversal on the first (and only) discrimination problem. Either trials or errors to criterion can be used; $\mathrm{RI}$ is calculated by dividing the reversal performance score by the acquisition performance score.

By way of contrast, $R / A$ is based on fixed-trial, rather than criterional, acquisition and reversal performances. A relatively small number of trials is provided for acquisition (e.g., 7, 9, or 11 trials on a predetermined basis from problem to problem) and for reversal (e.g., eight trials) for a series of discrimination problems (perhaps as many as 100) where a different pair of objects is used for each problem. The $\mathrm{R} / \mathrm{A}$ ratio is calculated for each of several blocks of problems (e.g., 20 problems), dividing percentage responses correct on reversal trials by percentage responses correct on acquisition trials. The ratios are then plotted by groups of $\mathrm{Ss}$ with $\mathrm{R} / \mathrm{A}$ ratio values on the ordinate and $A \%$ values from 50 to 100 on the abscissa. Thus, the A\% value (percentage responses correct on acquisition trials) enters twice into the assessment-in calculation of the $R / A$ ratio and then in classification of the ratio according to proficiency of prereversal performance (i.e., A\%). The first use 
of the $\mathrm{A} \%$ value is used to translate reversal performance to a value relative to acquisition performance; the second use of it classifies that translation of the reversal performance, i.e., the $\mathrm{R} / \mathrm{A}$ ratio, according to whether the $S$ was doing poorly or well prior to reversal trials.

The RI authors believe that their ratio is relatively insensitive to problem difficulty. They have reported that the $R I$ ratio remained essentially unchanged in two different training situations shown to be considerably different in difficulty. The $\mathrm{R} / \mathrm{A}$ authors handle what they view as a basic variable-differences in task difficulty-by graphically plotting and evaluating the $R / A$ ratios by $A \%$ values. The $R / A$ ratios are directly compared only where problem difficulty is known to be equivalent, an implication indexed by the A\% values.

A relatively minor point of difference is apparent in use of trial 1 acquisition and trial 1 reversal data. Whereas they are included in RI, probably without effect as the number of trials involved is relatively large, they are excluded in $\mathrm{R} / \mathrm{A}$ where performances are transformed in percentages and relatively more trial 1s are involved.

Both RI and R/A can be calculated and evaluated for individual $\mathrm{Ss}$, though groups of $\mathrm{Ss}$ are required for enhancement of reliability of each index and always required, of course, where generalizations to parent populations are desired.

2. Closely related to the immediately foregoing discussion is the point that RI entails calculation of a single ratio whereas $R / A$ entails calculation of as many ratios as there are blocks of problems administered to each $S$. The former provides for determining an index for organisms low in the phylogenetic scale to which it is impossible to administer a long series of problems during their life span. The latter provides for determining an $\mathrm{R} / \mathrm{A}$ function which changes with either continued training or with an increment in $\mathrm{A} \%$ or with varying levels of problem difficulty, however one chooses to perceive the matter at this point. With high organisms and particularly with primates, the calculation of many ratios entailed by the use of $R / A$ doubtless increases the intrinsic reliability of this index and favors its use over that of RI.

3. As RI is based on criterional acquisition and reversal, efficient reversal performances yield small number of trial (or error) scores which, in turn, yield small ratios which approach 0.00 . By contrast, as $\mathrm{R} / \mathrm{A}$ is based on percentages for responses correct, efficient reversal performances yield high $R \%$ values and, correspondingly, yield ratios larger than 1.00 (when $A \%$ is relatively low), but never greater than 2.00. The ceiling of 2.00 for the $R / A$ ratio is determined by the simple facts that $R$ can never exceed $100 \%$ and $A$ can never reliably be less than 50\%. Again, by contrast, RI can yield ratios ranging from fractions of 1.00 to infinity, theoretically, as some Ss might learn to a criterion but never be able to reverse to a criterion. Where reversal performance is efficient, relative to acquisition performance, $R I$ approaches 0.00 and R/A approaches 2.00; where reversal performance is in efficient, relative to acquisition performance, RI approaches infinity and R/A approaches 0.00 .

\section{The Inter-relatedness of $R I$ and $R / A$}

As viewed by the authors, points of similarity and dissimilarity between RI and R/A indicate an important relationship. Both indices are obtained from Ss initially naive to discrimination reversal training situations, and it is in reference to this point that the two indices are best integrated, perhaps. Surely there are many forms of life which by reason of either their phyletically-limited learning ability, age, brain damage, and the like, simply cannot learn in a discrimination-reversal training situation where a small and fixed number of trials are given pre- and post-reversal of cue values. On the other hand, it is clear that there are other animals that do learn under these conditions, most notably the primates. In the former instance it would be possible to obtain RI, perhaps, but impossible to obtain R/A. In the latter instance, whereas it would be possible to obtain both indices, for reasons of reliability, multiple ratios per $S$ within groups, and definition of $R / A$ functions by groups according to mastery levels and/or problem difficulty levels (A\% values), R/A offers possible advantage over RI.

Empirical tests to evaluate the kinds of problems and Ss for most effective use of the $R I$ and $R / A$ indices are, of course, possible. Certain of these tests are in advanced stages of investigation by the authors of this paper. Until results from them are available in form for dissemination, it is hoped that the present paper will serve to clarify the RI and R/A indices and to stimulate work toward the end of devising methods of measurement to provide for defensible comparisons of disparate organisms, necessarily trained in different training situations as truly equivalent situations are often beyond conception.

\section{References}

Harlow, H. F. Learning set and error factor theory. In S. Koch (Ed.), Psychology: a study of a science. Vol. 2. New York: McGraw-Hill, 1959. Pp. 492-537.

Rajalakshmi, R., \& Jeeves, M. A. The relative difficulty of reversal learning (reversal index) as a basis of behavioural comparisons. Anim. Behav., 1965, 8, 203-211.

Rumbaugh, D. M., \& Pournelle, M. B. Discrimination-reversal skills of primates: the reversal/acquisition ratio as a function of phyletic standing. Psychon. Sci., 1966, 4, 45-46.

\section{Note}

1. Work of this author supported by the National science Foundation. The counsel of Austin H. Riesen is gratefully acknowledged. 